\title{
无溶剂条件下简单、有效的合成多取代环己醇
}

\author{
荣良策*,a,b魏贤勇*,a 路 瑶 ${ }^{a}$ 宗志敏 ${ }^{a}$ \\ $\left({ }^{a}\right.$ 中国矿业大学化工学院＼cjkstart徐州 221008) \\ ( ${ }^{b}$ 江苏师范大学化学化工学院 徐州 221116)
}

\begin{abstract}
摘要 取代苯乙酮与查尔酮在氢氧化钠存在下于 $70{ }^{\circ} \mathrm{C}$ 加热反应, 取代苯乙酮与两分子查尔酮发生加成和环化反应, 生成了多取代环已醇. 本方法具有反应时间短、容易操作、产率高和环境友好等优点. 产物的结构经过红外、核磁和 元素分析确定，无溶剂方法是合成此类化合物的一条有效途径.

关键词 查尔酮; 环已醇; 无溶剂反应; 迈克尔加成反应; 羟醛缩合
\end{abstract}

\section{A Facile and Efficient Synthesis of Polysubstituted Cyclohex- anol Derivatives under Solvent-Free Conditions}

\author{
Rong, Liangce ${ }^{*, a, b} \quad$ Wei, Xianyong ${ }^{*, a} \quad$ Lu, Yao $^{a} \quad$ Zong, Zhimin ${ }^{a}$ \\ $\left({ }^{a}\right.$ School of Chemical Engineering and Technology, China University of Mining and Technology, Xuzhou 221008) \\ ( ${ }^{b}$ College of Chemistry and Chemical Engineering, Jiangsu Normal University, Xuzhou 221116)
}

\begin{abstract}
A facile and efficient synthesis of polysubstituted cyclohexanol derivatives by the addition and cyclization reaction of substituted acetophenone and chalcone in the presence of $\mathrm{NaOH}$ under solvent-free conditions is reported. This protocol has the advantages of shorter reaction time, mild conditions, easy work-up, and environmental friendliness. The products were identified by IR, ${ }^{1} \mathrm{H}$ NMR and elemental analysis. The solvent-free method is the efficient approach for the synthesis of these compounds.
\end{abstract}

Keywords chalcone; cyclohexanol; solvent-free reaction; Michael addition; aldol condensations

无溶剂条件下的有机合成由于在反应过程中不使 用或反应后期使用极少量的溶剂纯化产物，所以容易实 施和操作而引起人们极大关注，并得到了迅速的发展， 近年来有大量的无溶剂反应的文献 [1 3] 报道. 而且无 溶剂有机反应还可以有效的避免因使用溶剂所带来的 环境污染, 又可提高原料的利用率, 具有操作安全、简 单, 后处理方便等优点, 是实现绿色合成的一种重要方 法和手段. 更让人们感兴趣的是无溶剂合成还可以常常 产生一些在液相条件下难以形成的化合物. 因此无溶剂 条件下的有机反应为合成有机化合物提供更多、更简 单、更为有效的方法.

环己醇是重要的化工原料, 工业上或实验室中常用 来合成己二酸、己二胺、环己酮、己内酰胺等化工产品,
还可用于制备不饱和聚酯树脂、增塑剂、油漆、涂料等 重要大分子化合物 ${ }^{[4]}$, 这类化合物的合成一直受到人们 的关注. 查尔酮由于分子结构中含有碳碳双键和碳氧双 键两个活性中心，并且二者可以相互影响，是发生 Michael 加成 ${ }^{[5]}$, Knoevenagel 缩合 ${ }^{[6]}$ 和分子内 Aldol 反应 的重要底物, 在有机合成中具有广泛的应用. 文献报道 一些活性亚甲基化合物可以和查尔酮发生 Michael 加成 和分子内 Aldol 反应生成环已醇. 例如丙二睛 ${ }^{[7]}$ 、氧乙酸 乙酯 ${ }^{[8]}$ 和硝基甲烷 ${ }^{[9]}$ 都可以和查尔酮反应生成相应的环 己醇衍生物. 本文将报道取代苯乙酮与查尔酮在无溶剂 条件下也可发生 Michael 加成和分子内 Aldol 反应生成 多取代环已醇衍生物. 反应如 Eq. 1 所示.

\footnotetext{
*E-mail: 1crong@jsnu.edu.cn or lcrong2005@yahoo.com

Received April 5, 2012; revised May 15, 2012; published online June 4, 2012.

Project supported by the Natural Science Foundation of Jiangsu Education Department (No. 08KJB150017) and the Foundation of Xuzhou Normal University (No. 10XLS02).

江苏省高校自然科学基础研究基金(No. 08KJB150017)和江苏师范大学科研平台(No. 10XLS02)资助项目.
} 


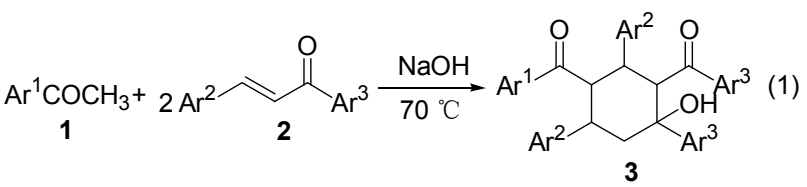

3a: $\mathrm{Ar}^{1}=\mathrm{C}_{6} \mathrm{H}_{5}, \mathrm{Ar}^{2}=\mathrm{C}_{6} \mathrm{H}_{5}, \mathrm{Ar}^{3}=\mathrm{C}_{6} \mathrm{H}_{5} ; \mathbf{3 b}: \mathrm{Ar}^{1}=\mathrm{C}_{6} \mathrm{H}_{5}, \mathrm{Ar}^{2}=4-\mathrm{FC}_{6} \mathrm{H}_{4}$, $\mathrm{Ar}^{3}=\mathrm{C}_{6} \mathrm{H}_{5} ; 3 \mathbf{c}: \mathrm{Ar}^{1}=\mathrm{C}_{6} \mathrm{H}_{5}, \mathrm{Ar}^{2}=2-\mathrm{ClC}_{6} \mathrm{H}_{4}, \mathrm{Ar}^{3}=\mathrm{C}_{6} \mathrm{H}_{5} ; 3 \mathbf{d}: \mathrm{Ar}^{1}=$ $\mathrm{C}_{6} \mathrm{H}_{5}, \mathrm{Ar}^{2}=4-\mathrm{ClC}_{6} \mathrm{H}_{4}, \mathrm{Ar}^{3}=\mathrm{C}_{6} \mathrm{H}_{5} ; 3 \mathbf{e}: \mathrm{Ar}^{1}=\mathrm{C}_{6} \mathrm{H}_{5}, \mathrm{Ar}^{2}=4-\mathrm{CH}_{3} \mathrm{C}_{6} \mathrm{H}_{4}$, $\mathrm{Ar}^{3}=\mathrm{C}_{6} \mathrm{H}_{5} ; 3 \mathbf{3}: \mathrm{Ar}^{1}=3-\mathrm{ClC}_{6} \mathrm{H}_{4}, \mathrm{Ar}^{2}=\mathrm{C}_{6} \mathrm{H}_{5}, \mathrm{Ar}^{3}=3-\mathrm{ClC}_{6} \mathrm{H}_{4} ; \mathbf{3 g}: \mathrm{Ar}^{1}=$ 4- $\mathrm{ClC}_{6} \mathrm{H}_{4}, \mathrm{Ar}^{2}=\mathrm{C}_{6} \mathrm{H}_{5}, \mathrm{Ar}^{3}=4-\mathrm{ClC}_{6} \mathrm{H}_{4} ; 3 \mathrm{~h}: \mathrm{Ar}^{1}=4-\mathrm{CH}_{3} \mathrm{C}_{6} \mathrm{H}_{4}, \mathrm{Ar}^{2}=$ $4-\mathrm{CH}_{3} \mathrm{C}_{6} \mathrm{H}_{4}, \quad \mathrm{Ar}^{3}=4-\mathrm{CH}_{3} \mathrm{C}_{6} \mathrm{H}_{4} ; \quad 3 \mathrm{i}: \quad \mathrm{Ar}^{1}=4-\mathrm{CH}_{3} \mathrm{C}_{6} \mathrm{H}_{4}, \quad \mathrm{Ar}^{2}=$ 3,4- $\left(\mathrm{CH}_{3}\right)_{2} \mathrm{C}_{6} \mathrm{H}_{3}, \mathrm{Ar}^{3}=4-\mathrm{CH}_{3} \mathrm{C}_{6} \mathrm{H}_{4} ; 3 \mathbf{j}: \mathrm{Ar}^{1}=4-\mathrm{CH}_{3} \mathrm{OC}_{6} \mathrm{H}_{4}, \mathrm{Ar}^{2}=\mathrm{C}_{6} \mathrm{H}_{5}$, $\mathrm{Ar}^{3}=4-\mathrm{CH}_{3} \mathrm{OC}_{6} \mathrm{H}_{4}$

\section{1 实验部分}

\section{1 仪器和试剂}

玛瑙研针; 熔点测定使用北京科仪电光仪器厂生产 的 TX5 显微熔点仪测定(温度未经校正); 红外光谱采用 FT/IR-8101 型红外光谱仪测定(KB 压片); 核磁共振氢 谱采用 Bruker-400 MHz 型核磁共振仪测定, DMSO- $d_{6}$ 为溶剂, TMS 为内标; 元素分析用 Perkin-Elmer 2400II 型元素分析仪测定. 所用试剂均为分析纯或化学纯, 使 用前未经纯化. 查尔酮按文献[10]方法合成.

\section{2 多取代环己醇衍生物的合成}

取代苯乙酮 $(1.5 \mathrm{mmol})$, 查尔酮 $(3 \mathrm{mmol})$ 和 $\mathrm{NaOH}$ (25 mmol) 置于玛瑙研钵中混合均匀, 反应物迅速转移 到 $50 \mathrm{~mL}$ 圆底烧瓶中, $70{ }^{\circ} \mathrm{C}$ 下反应约 $30 \sim 60 \mathrm{~min}$, 得黄 色固体, $\mathrm{HCl}$ 水溶液 (5\%)中和, 用水洗至中性, 抽滤, 固 体粗产物用 $95 \%$ EtOH 重结晶、干燥, 得白色固体 3.

1,3,5-三苯基-2,4-二苯甲酰基-1-环已醇(3a): 白色 固体, 收率 86\%. m.p. $242 \sim 243{ }^{\circ} \mathrm{C} ;{ }^{1} \mathrm{H}$ NMR (DMSO- $d_{6}$, $400 \mathrm{MHz}) \delta: 1.81(\mathrm{t}, J=10.0 \mathrm{~Hz}, 1 \mathrm{H}, \mathrm{CH}), 2.86(\mathrm{~s}, 1 \mathrm{H}$, $\mathrm{CH}), 3.73$ (s, 1H, CH), 3.95 (t, J=11.2 Hz, 1H, CH), 4.77 (d, $J=10.8 \mathrm{~Hz}, 1 \mathrm{H}, \mathrm{CH}), 5.04(\mathrm{~d}, J=11.6 \mathrm{~Hz}, 1 \mathrm{H}, \mathrm{CH})$, $5.16(\mathrm{~s}, 1 \mathrm{H}, \mathrm{OH}), 7.06 \sim 7.15(\mathrm{~m}, 11 \mathrm{H}, \mathrm{ArH}), 7.23 \sim 7.46$ (m, 12H, ArH), 7.63 (d, $J=7.6 \mathrm{~Hz}, 2 \mathrm{H}, \mathrm{ArH})$; IR (KBr) $v$ : 3408, 3060, 3028, 1669, 1646, 1596, 1579, 1559, 1541, 1493, 1448, 1396, 1346, 1255, 1220, 1181, 1067, 1002, 974, 953, 755, $699 \mathrm{~cm}^{-1}$. Anal. calcd for $\mathrm{C}_{38} \mathrm{H}_{32} \mathrm{O}_{3}: \mathrm{C}$ 85.05, H 6.01; found C 85.28, H 5.99.

1-苯基-3,5-二对氟苯基-2,4-二苯甲酰基-1-环己醇 (3b): 白色固体, 收率 83\%. m.p. $217 \sim 219{ }^{\circ} \mathrm{C} ;{ }^{1} \mathrm{H}$ NMR (DMSO- $\left.d_{6}, 400 \mathrm{MHz}\right) \delta: 1.82(\mathrm{dd}, J=3.2,13.2 \mathrm{~Hz}, 1 \mathrm{H}$, $\mathrm{CH}), 2.83\left(\mathrm{~d}, J=13.2 \mathrm{~Hz}, 1 \mathrm{H}, \mathrm{CH}_{3}\right), 3.70 \sim 3.77(\mathrm{~m}, 1 \mathrm{H}$, $\mathrm{CH}), 3.96(\mathrm{t}, J=11.2 \mathrm{~Hz}, 1 \mathrm{H}, \mathrm{CH}), 4.76(\mathrm{t}, J=11.2 \mathrm{~Hz}$, $1 \mathrm{H}, \mathrm{CH}), 5.01(\mathrm{~d}, J=11.2 \mathrm{~Hz}, 1 \mathrm{H}, \mathrm{CH}), 5.22(\mathrm{~s}, 1 \mathrm{H}, \mathrm{OH})$, $6.66(\mathrm{t}, J=9.2 \mathrm{~Hz}, 2 \mathrm{H}, \mathrm{ArH}), 6.92 \sim 6.99(\mathrm{~m}, 3 \mathrm{H}, \mathrm{ArH})$, $7.11 \sim 7.21(\mathrm{~m}, 7 \mathrm{H}, \mathrm{ArH}), 7.29 \sim 7.45(\mathrm{~m}, 9 \mathrm{H}, \mathrm{ArH}), 7.64$ (d, $J=7.6 \mathrm{~Hz}, 2 \mathrm{H}, \mathrm{ArH})$; IR (KBr) v: 3297, 3058, 2930, 1661, 1599, 1580, 1509, 1447, 1416, 1389, 1300, 1256, 1223, 1182, 1158, 1091, 1069, 1003, 971, 951, 891, 834, $785,761,710,692,667,562,537,525 \mathrm{~cm}^{-1}$. Anal. calcd for $\mathrm{C}_{38} \mathrm{H}_{30} \mathrm{~F}_{2} \mathrm{O}_{3}$ : C 79.70, H 5.28; found C 79.55, H 5.32.

1-苯基-3,5-二邻氯苯基-2,4-二苯甲酰基-1-环己醇 (3c): 白色固体, 收率 $80 \%$. m.p. $246 \sim 248{ }^{\circ} \mathrm{C}$ (lit. ${ }^{[11]}$ $208 \sim 210{ }^{\circ} \mathrm{C}$ ); ${ }^{1} \mathrm{H}$ NMR (DMSO- $\left.d_{6}, 400 \mathrm{MHz}\right) \delta: 1.77$ (d, $J=10.4 \mathrm{~Hz}, 1 \mathrm{H}, \mathrm{CH}), 2.63$ (s, 1H, CH), $4.46(\mathrm{~s}, 1 \mathrm{H}, \mathrm{CH})$, $4.67(\mathrm{~s}, 1 \mathrm{H}, \mathrm{CH}), 4.90(\mathrm{~d}, J=11.2 \mathrm{~Hz}, 1 \mathrm{H}, \mathrm{CH}), 5.07(\mathrm{~d}$, $J=11.6 \mathrm{~Hz}, 1 \mathrm{H}, \mathrm{CH}), 5.28(\mathrm{~s}, 1 \mathrm{H}, \mathrm{OH}), 6.68(\mathrm{~d}, J=7.6$ $\mathrm{Hz}, 1 \mathrm{H}, \mathrm{ArH}), 6.83 \sim 7.01(\mathrm{~m}, 4 \mathrm{H}, \mathrm{ArH}), 7.13 \sim 7.21(\mathrm{~m}$, 9H, ArH), 7.31 (t, $J=7.2 \mathrm{~Hz}, 2 \mathrm{H}, \operatorname{ArH}), 7.43$ (d, $J=7.2$ $\mathrm{Hz}, 2 \mathrm{H}, \mathrm{ArH}), 7.50$ (d, $J=7.6 \mathrm{~Hz}, 2 \mathrm{H}, \mathrm{ArH}), 7.64$ (d, $J=$ $8.0 \mathrm{~Hz}, 2 \mathrm{H}, \mathrm{ArH}), 7.90$ (d, $J=7.6 \mathrm{~Hz}, 1 \mathrm{H}, \mathrm{ArH})$; IR (KBr) $v: 3467,3057,2910,1673,1652,1595,1577,1559,1541$, 1492, 1476, 1447, 1386, 1299, 1255, 1218, 1193, 1160, 1134, 1036, 1001, 974, 957, 923, 755, 738, $695 \mathrm{~cm}^{-1}$. Anal. calcd for $\mathrm{C}_{38} \mathrm{H}_{30} \mathrm{Cl}_{2} \mathrm{O}_{3}$ : C 75.37, $\mathrm{H}$ 4.99; found $\mathrm{C}$ 75.62, H 4.96.

1-苯基-3,5-二对氯苯基-2,4-二苯甲酰基-1-环己醇 (3d): 白色固体, 收率 $87 \%$. m.p. $243 \sim 244{ }^{\circ} \mathrm{C}$ (lit. ${ }^{[1]}$ $187 \sim 189{ }^{\circ} \mathrm{C}$ ); ${ }^{1} \mathrm{H}$ NMR (DMSO- $\left.d_{6}, 400 \mathrm{MHz}\right) \delta: 1.80(\mathrm{~d}$, $J=10.4 \mathrm{~Hz}, 1 \mathrm{H}, \mathrm{CH}), 2.85$ (s, 1H, CH), $3.71(\mathrm{~s}, 1 \mathrm{H}, \mathrm{CH})$, $3.95(\mathrm{~s}, 1 \mathrm{H}, \mathrm{CH}), 4.77(\mathrm{~s}, 1 \mathrm{H}, \mathrm{CH}), 4.99(\mathrm{~d}, J=11.6 \mathrm{~Hz}$, $1 \mathrm{H}, \mathrm{CH}), 5.21(\mathrm{~s}, 1 \mathrm{H}, \mathrm{OH}), 6.90 \sim 6.98(\mathrm{~m}, 3 \mathrm{H}, \mathrm{ArH})$, $7.09 \sim 7.22(\mathrm{~m}, 9 \mathrm{H}, \mathrm{ArH}), 7.29 \sim 7.42(\mathrm{~m}, 9 \mathrm{H}, \mathrm{ArH}), 7.61$ (d, $J=7.2 \mathrm{~Hz}, 2 \mathrm{H}, \mathrm{ArH})$; IR (KBr) v: 3393, 3060, 3027, 1668, 1634, 1596, 1578, 1559, 1541, 1507, 1492, 1448, $1412,1346,1317,1256,1219,1181,1093,1071,1014$ 1001, 973, 954, 823, 783, 760, 731, 692, $668 \mathrm{~cm}^{-1}$. Anal. calcd for $\mathrm{C}_{38} \mathrm{H}_{30} \mathrm{Cl}_{2} \mathrm{O}_{3}$ : C 75.37, $\mathrm{H} 4.99$; found $\mathrm{C} 75.20, \mathrm{H}$ 5.00 .

1-苯基-3,5-二对甲苯基-2,4-二苯甲酰基-1-环己醇 (3e): 白色固体, 收率 $89 \%$. m.p. $262 \sim 264{ }^{\circ} \mathrm{C}$ (lit. ${ }^{[1]]}$ $197 \sim 198{ }^{\circ} \mathrm{C}$ ); ${ }^{1} \mathrm{H}$ NMR (DMSO- $\left.d_{6}, 400 \mathrm{MHz}\right) \delta: 1.76$ (dd, $J=3.6,13.6 \mathrm{~Hz}, 1 \mathrm{H}, \mathrm{CH}), 1.87$ (s, $\left.3 \mathrm{H}, \mathrm{CH}_{3}\right), 2.08$ (s, $\left.3 \mathrm{H}, \mathrm{CH}_{3}\right), 2.80(\mathrm{t}, J=13.2 \mathrm{~Hz}, 1 \mathrm{H}, \mathrm{CH}), 3.68(\mathrm{t}, J=9.6$ $\mathrm{Hz}, 1 \mathrm{H}, \mathrm{CH}), 3.90$ (t, $J=11.6 \mathrm{~Hz}, 1 \mathrm{H}, \mathrm{CH}), 4.72$ (t, $J=$ $11.2 \mathrm{~Hz}, 1 \mathrm{H}, \mathrm{CH}), 4.99$ (d, J=11.6 Hz, 1H, CH), 5.11 (s, $1 \mathrm{H}, \mathrm{OH}), 6.63(\mathrm{~d}, J=7.6 \mathrm{~Hz}, 2 \mathrm{H}, \mathrm{ArH}), 6.89 \sim 6.96(\mathrm{~m}$, $3 \mathrm{H}, \mathrm{ArH}), 7.08 \sim 7.17(\mathrm{~m}, 7 \mathrm{H}, \mathrm{ArH}), 7.21(\mathrm{~d}, J=8.0 \mathrm{~Hz}$, $3 \mathrm{H}, \mathrm{ArH}), 7.27 \sim 7.31(\mathrm{~m}, 2 \mathrm{H}, \mathrm{ArH}), 7.39$ (d, $J=8.0 \mathrm{~Hz}$, $4 \mathrm{H}, \mathrm{ArH}), 7.61$ (d, $J=7.6 \mathrm{~Hz}, 2 \mathrm{H}, \mathrm{ArH})$; IR (KBr) $v: 3477$, 3056, 3023, 2920, 1668, 1596, 1579, 1514, 1492, 1448, 
1384, 1349, 1309, 1256, 1221, 1191, 1095, 1069, 1002, $975,953,889,816,782,760,696,586 \mathrm{~cm}^{-1}$. Anal. calcd for $\mathrm{C}_{40} \mathrm{H}_{36} \mathrm{O}_{3}$ : C 85.07, H 6.43; found C 85.42, H 6.40.

1-间氯苯基-3,5-二苯基-2,4-二间氯苯甲酰基-1-环 己醇(3f): 白色固体, 收率 $80 \%$. m.p. $>280{ }^{\circ} \mathrm{C} ;{ }^{1} \mathrm{H}$ NMR (DMSO- $\left.d_{6}, 400 \mathrm{MHz}\right) \delta: 1.86(\mathrm{~d}, J=10.0 \mathrm{~Hz}, 1 \mathrm{H}, \mathrm{CH})$, $2.94(\mathrm{t}, J=13.2 \mathrm{~Hz}, 1 \mathrm{H}, \mathrm{CH}), 3.68(\mathrm{~s}, 1 \mathrm{H}, \mathrm{CH}), 3.97$ (t, $J=11.6 \mathrm{~Hz}, 1 \mathrm{H}, \mathrm{CH}), 4.70$ (d, $J=11.2 \mathrm{~Hz}, 1 \mathrm{H}, \mathrm{CH}), 4.97$ (d, $J=11.2 \mathrm{~Hz}, 1 \mathrm{H}, \mathrm{CH}), 5.43(\mathrm{~s}, 1 \mathrm{H}, \mathrm{OH}), 6.73 \sim 6.80(\mathrm{~m}$, $3 \mathrm{H}, \mathrm{ArH}), 6.86 \sim 6.91(\mathrm{~m}, 3 \mathrm{H}, \mathrm{ArH}), 6.99 \sim 7.10(\mathrm{~m}, 6 \mathrm{H}$, ArH), $7.12 \sim 7.41(\mathrm{~m}, 6 \mathrm{H}, \operatorname{ArH}), 7.52(\mathrm{~d}, J=8.0 \mathrm{~Hz}, 2 \mathrm{H}$, $\mathrm{ArH}), 7.71$ (d, $J=11.6 \mathrm{~Hz}, 2 \mathrm{H}, \mathrm{ArH})$; IR (KBr) $v: 3433$, 3065, 3029, 1672, 1616, 1571, 1494, 1473, 1455, 1422, 1318, 1249, 1216, 1194, 1073, 999, 889, 784, 771, 759, $700,671 \mathrm{~cm}^{-1}$. Anal. calcd for $\mathrm{C}_{38} \mathrm{H}_{29} \mathrm{Cl}_{3} \mathrm{O}_{3}: \mathrm{C} 71.31, \mathrm{H}$ 4.57; found C 71.13, H 4.66.

1-对氯苯基-3,5-二苯基-2,4-二对氯苯甲酰基-1-环 己醇(3g): 白色固体, 收率 82\%. m.p. 195 197 ${ }^{\circ} \mathrm{C} ;{ }^{1} \mathrm{H}$ NMR (DMSO- $\left.d_{6}, 400 \mathrm{MHz}\right) \delta: 1.82(\mathrm{~d}, J=6.0 \mathrm{~Hz}, 1 \mathrm{H}$, $\mathrm{CH}), 2.88(\mathrm{~s}, 1 \mathrm{H}, \mathrm{CH}), 3.69$ (s, 1H, CH), 3.94 (t, $J=11.6$ $\mathrm{Hz}, 1 \mathrm{H}, \mathrm{CH}), 4.69$ (d, $J=10.8 \mathrm{~Hz}, 1 \mathrm{H}, \mathrm{CH}), 4.95$ (d, $J=$ $11.6 \mathrm{~Hz}, 1 \mathrm{H}, \mathrm{CH}), 5.35(\mathrm{~s}, 1 \mathrm{H}, \mathrm{OH}), 6.75(\mathrm{t}, J=7.6 \mathrm{~Hz}$, $1 \mathrm{H}, \operatorname{ArH}), 6.89(\mathrm{t}, J=8.0 \mathrm{~Hz}, 2 \mathrm{H}, \operatorname{ArH}), 6.99(\mathrm{t}, J=7.6$ $\mathrm{Hz}, 1 \mathrm{H}, \mathrm{ArH}), 7.11 \sim 7.40$ (m, 16H, ArH), 7.61 (d, $J=8.4$ Hz, 2H, ArH); IR (KBr) v: 3446, 3061, 3029, 2917, 1669, $1589,1569,1490,1454,1400,1337,1254,1218,1176$, 1094, 1013, 975, 953, 860, 838, 795, 771, 756, 735, 700, $678 \mathrm{~cm}^{-1}$. Anal. calcd for $\mathrm{C}_{38} \mathrm{H}_{29} \mathrm{C}_{13} \mathrm{O}_{3}: \mathrm{C}$ 71.31, H 4.57; found C 71.59, H 4.48 .

1,3,5-三对甲基苯基-2,4-二对甲基苯甲酰基-1-环己 醇(3h): 白色固体, 收率 85\%. m.p. 227 228 ${ }^{\circ} \mathrm{C} ;{ }^{1} \mathrm{H}$ NMR (DMSO- $\left.d_{6}, 400 \mathrm{MHz}\right) \delta: 1.69(\mathrm{~d}, J=10.0 \mathrm{~Hz}, 1 \mathrm{H}$, $\mathrm{CH}), 2.08$ (s, $\left.6 \mathrm{H}, 2 \times \mathrm{CH}_{3}\right), 2.13\left(\mathrm{~s}, 3 \mathrm{H}, \mathrm{CH}_{3}\right), 2.18(\mathrm{~s}, 6 \mathrm{H}$, $\left.2 \times \mathrm{CH}_{3}\right), 2.68(\mathrm{~d}, J=6.0 \mathrm{~Hz}, 1 \mathrm{H}, \mathrm{CH}), 3.64(\mathrm{~s}, 1 \mathrm{H}, \mathrm{CH})$, 3.83 (s, 1H, CH), 4.69 (s, 1H, CH), 4.96 (d, $J=12.0 \mathrm{~Hz}$, $1 \mathrm{H}, \mathrm{CH}), 5.10(\mathrm{~s}, 1 \mathrm{H}, \mathrm{OH}), 6.10(\mathrm{~d}, J=7.6 \mathrm{~Hz}, 2 \mathrm{H}, \mathrm{ArH})$, $6.89 \sim 6.97$ (m, 10H, ArH), $7.18(\mathrm{~d}, J=7.6 \mathrm{~Hz}, 3 \mathrm{H}, \mathrm{ArH})$, 7.35 (t, $J=8.0 \mathrm{~Hz}, 4 \mathrm{H}, \mathrm{ArH}), 7.49$ (d, $J=8.0 \mathrm{~Hz}, 2 \mathrm{H}$, ArH); IR (KBr) v: 3448, 3029, 2917, 1654, 1607, 1567, 1512, 1412, 1380, 1330, 1308, 1287, 1255, 1225, 1207, 1181, 1116, 1029, 1015, 994, 828, 812, $735 \mathrm{~cm}^{-1}$. Anal. calcd for $\mathrm{C}_{43} \mathrm{H}_{42} \mathrm{O}_{3}$ : C 85.11, H 6.98; found $\mathrm{C} 85.30, \mathrm{H}$ 7.03 .

1-对甲基苯基-3,5-二(3,4-二甲基苯基)-2,4-二对甲 基苯甲酰基-1-环己醇(3i): 白色固体, 收率 80\%. m.p.
255 $257{ }^{\circ} \mathrm{C} ;{ }^{1} \mathrm{H}$ NMR (DMSO- $\left.d_{6}, 400 \mathrm{MHz}\right) \delta: 1.75$ (s, $1 \mathrm{H}, \mathrm{CH}), 1.83\left(\mathrm{~d}, J=9.6 \mathrm{~Hz}, 3 \mathrm{H}, \mathrm{CH}_{3}\right), 1.93 \sim 2.18(\mathrm{~m}$, $\left.15 \mathrm{H}, 5 \times \mathrm{CH}_{3}\right), 2.28(\mathrm{~s}, 1 \mathrm{H}, \mathrm{CH}), 2.41\left(\mathrm{~s}, 3 \mathrm{H}, \mathrm{CH}_{3}\right), 3.58$ (s, 1H, CH), $3.75(\mathrm{~s}, 1 \mathrm{H}, \mathrm{CH}), 4.72(\mathrm{~s}, 1 \mathrm{H}, \mathrm{CH}), 4.98(\mathrm{~d}$, $J=11.6 \mathrm{~Hz}, 1 \mathrm{H}, \mathrm{CH}), 5.12(\mathrm{~s}, 1 \mathrm{H}, \mathrm{OH}), 6.92 \sim 6.98(\mathrm{~m}$, $7 \mathrm{H}, \mathrm{ArH}), 7.35 \sim 7.40$ (m, 9H, ArH), 7.70 (d, $J=4.8 \mathrm{~Hz}$, $2 \mathrm{H}, \mathrm{ArH})$; IR (KBr) v: 3420, 3024, 2919, 1669, 1653, 1592, 1569, 1541, 1506, 1455, 1407, 1331, 1312, 1286, 1257, 1240, 1224, 1204, 1181, 1124, 1085, 1036, 1017, 988, 818, 723, $585 \mathrm{~cm}^{-1}$. Anal. calcd for $\mathrm{C}_{45} \mathrm{H}_{46} \mathrm{O}_{3}: \mathrm{C}$ 85.14, H 7.30; found C 85.28, H 7.32.

1-对甲氧基苯基-3,5-二苯基-2,4-二对甲氧基苯甲酰 基-1-环己醇(3j): 白色固体, 收率 84\%. m.p. 216 218 ${ }^{\circ} \mathrm{C} ;{ }^{1} \mathrm{H}$ NMR (DMSO- $\left.d_{6}, 400 \mathrm{MHz}\right) \delta: 1.73(\mathrm{~d}, J=10.0 \mathrm{~Hz}$, $1 \mathrm{H}, \mathrm{CH}), 2.73(\mathrm{t}, J=12.4 \mathrm{~Hz}, 1 \mathrm{H}, \mathrm{CH}), 3.60(\mathrm{~s}, 3 \mathrm{H}$, $\left.\mathrm{OCH}_{3}\right), 3.65$ (s, $\left.3 \mathrm{H}, \mathrm{OCH}_{3}\right), 3.67(\mathrm{~s}, 1 \mathrm{H}, \mathrm{CH}), 3.70(\mathrm{~s}, 3 \mathrm{H}$, $\left.\mathrm{OCH}_{3}\right), 3.85$ (t, $\left.J=11.2 \mathrm{~Hz}, 1 \mathrm{H}, \mathrm{CH}\right), 4.70$ (t, $J=10.8 \mathrm{~Hz}$, $1 \mathrm{H}, \mathrm{CH}), 4.96(\mathrm{~d}, J=11.6 \mathrm{~Hz}, 1 \mathrm{H}, \mathrm{CH}), 5.22(\mathrm{~s}, 1 \mathrm{H}, \mathrm{OH})$, $6.65 \sim 6.71(\mathrm{~m}, 7 \mathrm{H}, \mathrm{ArH}), 6.82(\mathrm{t}, J=7.6 \mathrm{~Hz}, 2 \mathrm{H}, \operatorname{ArH})$, 6.97 (t, $J=7.2 \mathrm{~Hz}, 1 \mathrm{H}, \mathrm{ArH}), 7.10(\mathrm{t}, J=7.6 \mathrm{~Hz}, 2 \mathrm{H}$, $\operatorname{ArH}), 7.31(\mathrm{~d}, J=7.6 \mathrm{~Hz}, 3 \mathrm{H}, \mathrm{ArH}), 7.42 \sim 7.55(\mathrm{~m}, 7 \mathrm{H}$, ArH); IR (KBr) v: 3382, 3061, 3029, 2934, 2838, 1662, $1600,1573,1511,1455,1421,1343,1309,1257,1228$, 1171, 1117, 1072, 1031, 974, 955, 866, 841, 783, 768, 737, $701,601 \mathrm{~cm}^{-1}$. Anal. calcd for $\mathrm{C}_{41} \mathrm{H}_{38} \mathrm{O}_{6}$ : C 78.57, H 6.11; found C 78.33, H 6.15.

\section{2 结果和讨论}

查尔酮属于 $\alpha, \beta$-不饱和酮, 分子中含有碳碳双键和 羰基的两种官能团, 较易和其他化学试剂发生加成、缩 合和氧化反应, 是有机合成常用的底物. 我们曾报道查 尔酮与环己䣯 ${ }^{[12]} 、 1$ - 荥䣯 ${ }^{[13]}$ 在室温研磨条件下很容易发 生迈克尔加成的反应生成相应的加成产物, 我们也想将 苯乙酮与查尔酾(1,3-二苯基-2-丙烯-1-酮)在室温下研 磨, 试图得到与上述相似的加成产物, 但是令我们遗憾 的是反应并没有发生，于是我们又尝试将反应混合物在 $70{ }^{\circ} \mathrm{C}$ 加热 $30 \mathrm{~min}$, 研究反应能否发生. 我们通过 TLC 检测发现有新的物质生成, 我们向反应体系加入 $5 \%$ 的 盐酸溶液中和反应混合物中碱, 发现有大量不溶于水物 质浮在水面上，通过分离、提纯，将得到的物质进行红 外、核磁测试, 我们发现生成的产物并不是苯乙酮与查 尔酮加成的产物, 而是另有其他物质生产, 经过仔细分 析图谱数据, 我们确定产物是苯乙酮与两分子查尔酮发 生了加成反应和分子内的 Aldol 反应所生成的五取代环 己醇的衍生物. 受此结果鼓舞, 我们想进一步研究体系 
反应情况. 我们将带有取代基的苯乙酮与不同的取代基 查尔酮放在一起加热反应，结果发现它们也是可以顺利 发生反应得到目标产物的. 我们同时发现查尔酮的芳环 上无论带有是吸电子基团如氟原子、氯原子, 还是带有 供电子基团如甲基、甲氧基, 对反应并没有明显的影响, 反应都可以顺利进行并以较高的收率得到多取代环己 醇衍生物. 尽管文献[10]也报道了相似化合物的合成, 但是它所报道的都是苯乙酮与醛反应的产物, 没有取代 苯乙酮参与的反应，我们报道的方法由于可以使用取代 苯乙酮与取代查尔酮之间进行反应，所以更有利于合成 含有更多取代基的环已醇产物。

反应的产物经过红外、核磁和元素分析确定. 例如 在红外光谱中 $3400 \mathrm{~cm}^{-1}$ 附近出现一个明显的尖峰, 应 该是产物中的羟基吸收峰, $1660 \mathrm{~cm}^{-1}$ 附近出现的较强 尖峰应该是分子中的羰基峰. 在核磁中, 以 $\mathbf{3 a}$ 为例, $\delta$ $1.81(\mathrm{t}, J=10.0 \mathrm{~Hz}, 1 \mathrm{H}, \mathrm{CH}), 2.86(\mathrm{~s}, 1 \mathrm{H}, \mathrm{CH}), 3.73(\mathrm{~s}$, $1 \mathrm{H}, \mathrm{CH}), 3.95$ (t, $J=11.2 \mathrm{~Hz}, 1 \mathrm{H}, \mathrm{CH}), 4.77$ (d, $J=10.8$ $\mathrm{Hz}, 1 \mathrm{H}, \mathrm{CH}), 5.04(\mathrm{~d}, J=11.6 \mathrm{~Hz}, 1 \mathrm{H}, \mathrm{CH})$ 等 6 个质子应 该是新形成的环己烷的环上的脂肪氢， $\delta 5.16(\mathrm{~s}, 1 \mathrm{H}$, $\mathrm{OH}$ )处的单峰应该是生成的羟基质子氢, 25 个芳氢也是 与产物的结构吻合. 我们推测产物的生成过程应该是取 代苯乙酮与取代查尔酮先发生双分子 Michael 加成反应, 然后发生分子内的 Aldol 缩合反应而完成的. 由于反应 过程不使用有机溶剂, 仅在最后产物纯化时使用极少量 的乙醇, 所以对环境影响较小. 本文为此类化合物提供
了一条简单、有效的绿色合成途径.

\section{References}

[1] Mehdi, A.; Ehsan, S.; Mehdi, B.; Hamid, R. B.; Masoud, A. Tetrahedron 2012, 68, 3237.

[2] Mazaahir, K.; Anwar, J.; Ritika, C.; Neeraj, K. M. Tetrahedron Lett. 2012, 53, 1728.

[3] Marcos, A. P. M.; Clarissa, P. F.; Dayse, N. M.; Lilian, B.; Pablo, M. Chem. Rev. 2009, 109, 4140.

[4] Zhang, L. F.; Chen, C. Y.; Xiang, Z. J. J. Beijing Insitute Petro-Chem. Technol. 2004, 12, 39 (in Chinese).

(张丽芳, 陈赤阳, 项志军, 北京石油化工学院学报, 2004, 12, 39.)

[5] Zhang, Z.; Dong, Y. W.; Wang, G. W.; Komatsu, K. Synlett 2004, 61.

[6] Moussa, H. H.; Chabaka, L. M. Egypt. J. Chem. 1983, 26, 267.

[7] Al-Arab, M. M.; Tabba, H. D.; Ghanem, B. S.; Olmstead, M. M. Synthesis 1990, 1157.

[8] Li, J. T.; Chen, G. F.; Yang, W. Z.; Li, T. S. Ultrason. Sonochem. 2003, 10,123

[9] Olivier, C.; Karine, G.; Jack, H.; Ludovic, P.; Framçoise, T. B.; Loïc, T. Tetrahedron Lett. 2004, 45, 391.

[10] Kohler, E. P.; Chadwell, H. M. Organic Synthesis: Collective, Vol. 1, John Willey \& Sons, New York, 1941, p. 78.

[11] Luo, X. X.; Shen, Z. X. Tetrahedron Lett. 2006, 47, 5623.

[12] Rong, L. C.; Li, X. Y.; Wang, H. Y.; Shi, D. Q.; Tu, S. J. Chin. J. Org. Chem. 2007, 27, 1292 (in Chinese).

(荣良策, 李小跃, 王海营, 史达清, 屠树江, 有机化学, 2007, 27, 1292.)

[13] Rong, L. C.; Han, H. X.; Jiang, H.; Li, X. Y.; Yao, C. S.; Tu, S. J. Chin. J. Org. Chem. 2008, 28, 746 (in Chinese).

(荣良策, 韩红霞, 蒋虹, 李小跃, 姚昌盛, 屠树江, 有机化学, 2008, 28, 746.) 